\title{
Development of Postpartum Depression in Pregnant Women with Preeclampsia: A Retrospective Study
}

\author{
Li Chen, ${ }^{1,2,3}$ Xiaodan Wang, ${ }^{1,2,3}$ Qian Ding, ${ }^{4}$ Nan Shan $\mathbb{D}^{1,2,3}$ and Hongbo Qi ${ }^{10}{ }^{1,2,3}$ \\ ${ }^{1}$ The Department of Obstetrics, The First Affiliated Hospital of Chongqing Medical University, Chongqing 400016, China \\ ${ }^{2}$ State Key Laboratory of Maternal and Fetal Medicine of Chongqing Municipality, Chongqing Medical University, \\ Chongqing 400016, China \\ ${ }^{3}$ International Collaborative Laboratory of Reproduction and Development of Chinese Ministry of Education, \\ Chongqing Medical University, Chongqing 400016, China \\ ${ }^{4}$ The Department of Dermatology and Venereology, The First Affiliated Hospital of Chongqing Medical University, \\ Chongqing 400016, China
}

Correspondence should be addressed to Nan Shan; shannancqmu@163.com and Hongbo Qi; qihongbocy@gmail.com

Received 5 November 2018; Revised 11 January 2019; Accepted 5 February 2019; Published 27 February 2019

Academic Editor: Marco Scioscia

Copyright (C) $2019 \mathrm{Li}$ Chen et al. This is an open access article distributed under the Creative Commons Attribution License, which permits unrestricted use, distribution, and reproduction in any medium, provided the original work is properly cited.

\begin{abstract}
Background. Postpartum depression (PPD) and preeclampsia (PE) are both common diseases in obstetrics that affect maternal health and infant development. However, the relationship between the two diseases still requires clarification. Objective. The purpose of this study was to (1) determine the incidence rate of PPD in patients with PE and (2) identify the association between the prevalence of PPD and the severity of PE. Methods. We conducted a retrospective analysis of women with and without PE who delivered between January 1, 2017, and August 30, 2018, in the First Affiliated Hospital of Chongqing Medical University. We used a questionnaire survey methodology that included the Edinburgh Postnatal Depression Scale (EPDS) to test the influence of PE on the development of new-onset PPD in the 6 weeks after delivery. We determined PPD based on a score $\geq 10$ on the EPDS. Bivariate analysis was used to compare data between the two groups. Results. A total of 180 women participated in this study. Thirty-five people screened positive for PPD, while the remaining 145 screened negative. The prevalence of PPD was 26.67\% (24/90) in patients with $\mathrm{PE}$, which was two times the prevalence in normal women (12.22\%). Multiple logistic regression showed that women who had PE had nearly 3 -fold increased odds of PPD compared to normal women and the risk of PPD increased with the aggravation of PE. Patients with severe PE had a more than 4 -fold increased risk of screening positive for PPD. Conclusion. PE was independently associated with PPD. Furthermore, the risk of PPD seemed to increase with the aggravation of PE. Thus, additional prevention efforts and support methods should be provided for women with PE to reduce the incidence of PPD.
\end{abstract}

\section{Introduction}

Puerperae experience pathophysiological changes and role shifts after childbirth and become more prone to depression than during other periods of life [1]. The incidence of postpartum depression (PPD) varies greatly among patients in different regions, of different races and with different economic backgrounds, with a prevalence range of approximately $6.5-19 \%[2,3]$. The morbidity is higher when women have cesarean section deliveries, are underweight, are obese, have pelvic floor symptoms, and experience other special situations [4-6]. PPD has a negative influence on women's health, family harmony, and infant development and has become a public health problem worldwide.

Preeclampsia (PE) is a common obstetric complication with an incidence of approximately $3-5 \%$ in all pregnant women [7]. At the same time, it is also a severe obstetric disease associated with serious maternal-fetal morbidity and mortality [8]. The pathogenesis of PE is still unclear and may be related to hypoperfusion and ischemia of the placenta. The ischemic placenta will cause oxidative stress and release inflammatory factors, thus leading to systemic endothelial dysfunction. The maternal consequences include disseminated intravascular coagulation, hypertensive 
encephalopathy, heart failure, thrombocytopenia, renal failure, and HELLP syndrome. Fetal consequences of PE include preterm labor, fetal distress, and fetal growth restriction [9].

Studies have shown that hypertension is a risk factor for depression; in hypertensive patients, the prevalence of depression is as high as $26.8 \%$ [10, 11]. Although chronic hypertension is slightly different from $\mathrm{PE}$ in patients in terms of pathogenesis and some clinical symptoms, the manifestations of both diseases are mainly elevated blood pressure and damage to vital organs, such as the heart, brain, and kidneys. However, it is unknown whether there is a link between PE and PPD, given that they are two common conditions in obstetrics. One study that investigated obstetric risk factors for depression mentioned that PE increased the incidence of PPD [12]. However, few articles have systematically researched the relationship between PE and PPD.

In our study, we collected data from eligible pregnant women who delivered between January 1, 2017, and August 30, 2018, in the First Affiliated Hospital of Chongqing Medical University. The purpose of this study was to (1) determine the incidence rate of PPD in patients with PE and (2) identify whether the prevalence of PPD will increase with the aggravation of $\mathrm{PE}$.

\section{Materials and Methods}

We obtained written informed consent from all subjects included in the research. We performed a retrospective study of women who delivered between January 1, 2017, and August 30, 2018, in the First Affiliated Hospital of Chongqing Medical University. All women were required to follow up in a specialty postpartum clinic approximately 6 weeks after delivery.

Women who met the inclusion criteria were included in our research. The inclusion criteria were as follows: (1) either being diagnosed with PE based on the clinical guidelines published by ACOG in 2013 [8] or not being diagnosed with $\mathrm{PE}$ and being part of the control group; (2) age between 20 to 40 years old; (3) gestational age $\geq 28$ weeks; and (4) vaginal delivery or cesarean section and neonatal survival. The exclusion criteria were as follows: (1) having obstetric complications other than PE; (2) having a previous history of PPD or anxiety, or a family history of depression, or other mental disorders; and (3) having a stillborn fetus or lethal induction of labor. Patients who met the inclusion and exclusion criteria were asked to fill in a questionnaire survey when they came to the specialty postpartum clinic approximately 6 weeks after delivery. The questionnaire survey collected information on the last 7 days and included graded questions (e.g., "What has been the range of your blood pressure fluctuations in the last 7 days?") and "yes/no" questions (e.g., "Have you taken any medicine to control your blood pressure in the last 7 days?"). Data on demographic characteristics, mode of delivery, and BMI before delivery were collected from the electronic medical records system in our institution.

The Edinburgh Postnatal Depression Scale (EPDS) is a self-evaluation scale that consists of 10 items. Each item is divided into 4 grades and is scored from 0 to 3 . The total score ranges from 0 to 30 , with higher scores signifying more serious PPD [13]. Based on the original scale, Chinese experts modified the language expression and choice format of each item to make the expression more consistent with the linguistic habits of the mainland Chinese population [14]. The content validity radio (CVR) of the Chinese edition of the EPDS is 0.9333 , and the internal consistency reliability is supported by Cronbach's $\alpha$ coefficient of 0.76 [14]. The coincidence rate of the EPDS for translation and backtranslation is $100 \%$ [14]. The EPDS is the most commonly used postnatal depression scale, used in $47.1 \%$ of healthcare facilities in China [15]. The proposed threshold for PPD in the Chinese edition is 9.5 points [14]. Therefore, a score on the EPDS $\geq 10$ was determined to be positive for PPD screening in our study.

All women included in our study were divided into PPD and NO-PPD groups according to the results of EPDS screening. The clinical data analysis was performed using SPSS 20.0 (IBM Corporation, Amon City, NY). If continuous variables conformed to a normal distribution, they were presented as the mean \pm standard deviation, and an independent sample ttest was used to determine the differences. Otherwise, continuous variables were described by the median \pm quartile, and the Mann-Whitney $U$ test was used to determine differences if the variables did not dissatisfy a normal distribution. Categorical data were expressed as frequencies and percentages, and chi-square tests were used to evaluate differences. $\mathrm{P}<0.05$ was considered to be statistically significant. Finally, multiple logistic regression was used to estimate crude odds ratios and adjusted odds ratios.

\section{Results}

A total of 90 patients with PE met the inclusion and exclusion criteria. We randomly selected 90 normal pregnant women who met the inclusion criteria in the same period. Finally, 180 women participated in our study and completed the questionnaire survey. They were divided into PPD and NOPPD groups according to whether they screened positive for EPDS; their data are summarized in Table 1. As shown in Table 1, a total of 35 people screened positive for PPD, while the remaining 145 screened negative. The differences in age, gestational weeks, gravidity, parity, prior number of cesarean sections, BMI before delivery, mode of delivery, premature birth, and neonatal weight were not significant between the two groups. However, in the PPD group, 24 women had $\mathrm{PE}$ with a much higher incidence rate than in the NO-PPD group, in which 66 women suffered from PE (24/35 vs. 66/145, $\mathrm{P}=0.014)$. Thus, $\mathrm{PE}$ may be a risk factor for PPD.

To further clarify whether the mode of delivery, gestational weeks, or demographic characteristics were risk factors for PPD, we analyzed these data in both normal women and patients with PE. Among the normal women, 11 suffered from PPD, with a prevalence of $12.22 \%$ (11/90). There was no significant difference between the two groups in demographic characteristics, mode of delivery, and premature birth rate. The collected data and bivariate analysis in women with and without PPD are shown in Table 2.

On the other hand, $26.67 \%$ (24/90) of patients with PE screened positive for PPD, which was two times more than the number of normal puerperae screening positive for 
TABLE 1: Comparison of demographic characteristics, delivery characteristics, and the proportion of preeclampsia in women with and without postpartum depression.

\begin{tabular}{lccc}
\hline Variables & PPD group $(\mathrm{N}=35)$ & NO-PPD group (N=145) & $P$ value \\
\hline Normal women & 11 & 66 & \\
Preeclampsia & 24 & 32 & 0.014 \\
Delivery mode & & 113 & \\
Vaginal delivery & 4 & $29(27-32)$ & 0.158 \\
Cesarean section & 31 & $2(1-3)$ & 0.621 \\
Age(y) & $30(27-33)$ & $0(0-1)$ & 0.905 \\
Gravidity & $2(1-4)$ & $0(0-0)$ & 0.516 \\
Parity & $0(0-1)$ & $271(261.5-277)$ \\
Previous C-S* & $0(0-0)$ & $3020(2740-3300)$ \\
Gestational weeks(d) & $268(262-275)$ & $28.23(26.04-30.32)$ \\
Neonatal weight $(\mathrm{g})$ & $2900(2350-3410)$ & & 0.810 \\
BMI $\left(\mathrm{kg} / \mathrm{m}^{2}\right)^{\#}$ & $28.34(25.78-29.34)$ & 33 \\
Premature birth & & 112 & 0.289 \\
Yes & 7 & 0.402 \\
No & 28 & 0.725 \\
\hline
\end{tabular}

Continuous variables are presented as the median \pm quartile, and the Mann-Whitney $\mathrm{U}$ test was used to determine differences. Categorical data are presented as frequencies, and the chi-square test was used to compare differences.

* Previous cesarean section.

\#BMI before delivery.

TABLE 2: Comparison of demographic characteristics and delivery characteristics in normal women.

\begin{tabular}{lccc}
\hline Variables & PPD group (N=11) & NO-PPD group (N=79) & $P$ value \\
\hline Delivery mode & & & \\
Vaginal delivery & 4 & 53 & 1.000 \\
Cesarean section & 7 & $30(27-32)$ & 0.456 \\
Age $(\mathrm{y})$ & $30(28-39)$ & $2(1-3)$ & 0.551 \\
Gravidity & $2(2-4)$ & $0(0-1)$ & 0.953 \\
Parity & $0(0-1)$ & $0(0-0)$ & 0.530 \\
Previous C-S* & $0(0-0)$ & $275(271-278)$ \\
Gestational weeks(d) & $273(268-276)$ & $3175(2950-3470)$ \\
Neonatal weight $(\mathrm{g})$ & $3290(2800-3400)$ & $27.56(26.22-28.89)$ & 0.452 \\
BMI $\left(\mathrm{kg} / \mathrm{m}^{2}\right)^{\#}$ & $28.35(24.80-29.34)$ & 0.530 \\
Premature birth & & 5 & 0.460 \\
Yes & 2 & 74 \\
No & 9 & 0.203 \\
\hline
\end{tabular}

Continuous variables are presented as the median \pm quartile, and the Mann-Whitney $\mathrm{U}$ test was used to determine differences. Categorical data are presented as frequencies, and the chi-square test was used to compare differences.

* Previous cesarean section.

\#BMI before delivery.

PPD. Among the 90 patients, 52 patients had severe PE. The incidence of PPD was $28.85 \%$ (15/52) in patients with severe PE. Five patients had slightly higher blood pressure in the previous 7 days, ranging from $140 / 90$ to $160 / 110 \mathrm{mmHg}$. Two of these patients screened positive for PPD, and 3 screened negative for PPD. There were 6 patients with PPD and 18 patients without PPD taking drugs (Adalat GITS) for blood pressure control in the previous 7 days. Only one patient with PE occasionally had mild headaches. Table 3 shows the basic data we collected for patients with PE. Similarly, there was no obvious difference in these data between the PPD group and NO-PPD group.

The average score of the EPDS (4.42 vs. 7.27, $P<$ 0.05 ) was obviously higher in patients with $\mathrm{PE}$ compared with normal women. When patients had severe PE, the average score was 8.65 with a significant difference to normal women (Table 4). Finally, we performed multiple logistic regression to estimate the adjusted odds ratio, with PPD as the dependent variable and the presence of PE, gravidity, parity, previous cesarean section, gestational weeks, neonatal weight, 
TABLE 3: Comparison of demographic characteristics and delivery characteristics in patients with preeclampsia.

\begin{tabular}{|c|c|c|c|}
\hline Variables & PPD group $(\mathrm{N}=24)$ & NO-PPD group $(\mathrm{N}=66)$ & $P$ value \\
\hline Severe preeclampsia & 15 & 37 & \\
\hline \multicolumn{4}{|l|}{ Blood pressure } \\
\hline$<140 / 90 \mathrm{mmHg}$ & 22 & 63 & \\
\hline 140/90 160/110 mmHg & 2 & 3 & \\
\hline$\geq 160 / 110 \mathrm{mmHg}$ & 0 & 0 & 0.862 \\
\hline \multicolumn{4}{|l|}{ Hypotensive } \\
\hline Yes & 6 & 18 & \\
\hline No & 18 & 48 & 0.829 \\
\hline \multicolumn{4}{|l|}{ Clinical symptoms } \\
\hline Yes & 0 & 1(headache) & \\
\hline No & 24 & 65 & 1.000 \\
\hline \multicolumn{4}{|l|}{ Mode of delivery } \\
\hline Vaginal delivery & 0 & 6 & \\
\hline Cesarean section & 24 & 60 & 0.293 \\
\hline $\operatorname{Age}(y)$ & $29(27-32.75)$ & $28.5(27-32.5)$ & 0.912 \\
\hline Gravidity & $2(1-3.75)$ & $2(1-3.25)$ & 0.771 \\
\hline Parity & $0(0-1)$ & $0(0-1)$ & 0.290 \\
\hline Previous C-S* & $0(0-1)$ & $0(0-1)$ & 0.812 \\
\hline Gestational weeks(d) & $265(262-274.25)$ & $263(251.75-270.75)$ & 0.361 \\
\hline Neonatal weight(g) & $2760(2220-3410)$ & $2825(2370-3210)$ & 0.729 \\
\hline $\operatorname{BMI}\left(\mathrm{kg} / \mathrm{m}^{2}\right)^{\#}$ & $26.64(25.81-30.03)$ & $29.28(25.98-31.44)$ & 0.085 \\
\hline \multicolumn{4}{|l|}{ Premature birth } \\
\hline Yes & 5 & 28 & \\
\hline No & 19 & 38 & 0.06 \\
\hline
\end{tabular}

Continuous variables are presented as the median \pm quartile, and the Mann-Whitney U test was used to determine differences. Categorical data are presented as frequencies, and the chi-square test was used to compare differences.

* Previous cesarean section

\#BMI before delivery.

TABLE 4: Comparison of EPDS scores between normal women and patients with preeclampsia.

\begin{tabular}{lcccc}
\hline Variables & Subjects $(\mathrm{n})$ & EPDS scores & $t$ test & $P$ value \\
\hline Normal women & 90 & $4.42 \pm 3.320$ & Reference & Reference \\
\hline Preeclampsia & 90 & $7.27 \pm 3.136$ & -4.178 & $P<0.05$ \\
\hline Severe preeclampsia & 52 & $8.65 \pm 3.610$ & -5.011 & $P<0.05$ \\
\hline
\end{tabular}

BMI before delivery, and mode of delivery as the independent variables (Table 5). The result showed that women who had PE had nearly 3-fold increased odds of PPD compared to normal women. When "severe PE" was used in the model, it had a more than 4 -fold increased risk for screening positive for PPD. This finding indicates that the risk of PPD will increase with the aggravation of $\mathrm{PE}$.

\section{Discussion}

To our knowledge, this retrospective study is the first to analyze the relationship between PE and PPD. In our study, the incidence of PPD was $19.44 \%$ (35/180), slightly higher than that reported in other articles [2, 3]. Among them, $26.67 \%$ of patients with PE screened positive for PPD, which was two times more than the number of normal women screening positive for PPD. With the exception of the postpartum state, people who suffer from hypertension have a higher risk of depression. A meta-analysis that included 41 articles with a total population of 30,796 suggested that the summarized incidence of depression among hypertensive patients is $26.8 \%$, which is similar to our finding [11].

Several mechanisms, such as clinical symptoms, inflammation, and genetic changes, have been used as hypotheses for the relationship between PE and PPD. In our study, although almost all women with $\mathrm{PE}$ had no obvious clinical symptoms when they completed the questionnaire survey, most of them suffered from headaches, cutaneous edema, and abnormal liver function several weeks before and after delivery. The clinical symptoms were more serious particularly in patients with severe PE. Of the 15 patients with severe PE and PPD, 10 had severe headaches, 7 had ascites before 
TABle 5: Multivariable logistic regression analysis for PPD.

\begin{tabular}{lcc}
\hline & Adjusted OR & $95 \%$ CI \\
\hline Age $(\mathrm{y})$ & 0.954 & $0.855-1.064$ \\
\hline Gravidity & 0.728 & $0.520-1.020$ \\
\hline Parity & 1.320 & $0.432-4.037$ \\
\hline Previous C-S* & 0.834 & $0.247-2.815$ \\
\hline Gestational weeks (d) & 1.018 & $0.970-1.068$ \\
\hline Neonatal weight $(\mathrm{g})$ & 0.999 & $0.998-1.000$ \\
\hline BMI $\left(\mathrm{kg} / \mathrm{m}^{2}\right)^{\#}$ & 1.200 & $1.023-1.406$ \\
\hline Preeclampsia & 2.753 & $1.056-7.178$ \\
\hline Severe preeclampsia & 4.540 & $1.194-17.255$ \\
\hline
\end{tabular}

delivery, and 5 had severe liver dysfunction with levels of serum transaminase more than 5 times higher than their normal levels. These symptoms persisted until approximately 2 weeks after delivery; they may have caused women persistent negative emotional experiences and thus become a trigger for PPD. Conversely, in patients who screened negative for PPD, the severity and duration of clinical symptoms associated with PE were less severe than in the PPD group. Therefore, clinical symptoms may be a hypothesis for the increasing incidence of PPD.

It is well known that the "two-stage theory" of the pathogenesis of $\mathrm{PE}$ has been proposed internationally in recent years. In the first stage, which occurs during the first trimester, vascular remodeling disorders of uterine spiral arterioles caused by multiple factors result in "superficial placental implantation" and ultimately cause insufficient placental perfusion and impairment of placental function. In the second stage, which occurs during the second and third trimesters, the ischemic placenta will experience oxidative stress and release inflammatory factors, thus leading to systemic endothelial dysfunction. Therefore, patients with PE often have excessive inflammation of the immune system and an increased number of inflammatory factors in blood circulation. Jonsson $\mathrm{Y}$ et al. measured more than 20 different kinds of cytokines in serum and found significant differences in IL- 6 and IL- 8 between normal pregnant women and subjects with PE [16]. Sharma A et al. performed a similar study and found that compared with normal pregnant women, the levels of TNF-alpha, IL-6, and IL-8 in patients with PE were significantly increased [17]. The study of Catarino C et al. also indicated a significant increase in levels of IL-6, TNF-alpha, and C-reactive protein in patients with PE [18]. In contrast, several articles have shown that elevated proinflammatory cytokines, such as high-sensitivity C-reactive protein and IL6 , have been associated with the development of PPD $[19,20]$. In addition, one study has noted that the development of depression and psychosis in young adulthood was associated with higher levels of IL-6 in childhood [21]. Therefore, increased inflammatory factors, such as C-reactive protein and IL-6, in the blood circulation of patients with PE may increase the incidence of PPD.

Finally, we attempted to explain the relationship between preeclampsia and PPD from the perspective of genetic changes since both of these diseases are caused by multiple factors and genes. A meta-analysis of 54 casecontrolled studies with a total of 7,398 patients with PE and 11,230 normal pregnant women found that the 5,10methylenetetrahydrofolate reductase (MTHFR) C677T genotype was a risk factor for PE [22]. Interestingly, PPD may also be accompanied by changes in the expression of certain genes, which has also been found in patients with PE. $\mathrm{Li}$ yin di et al. conducted a study on the relationship between PPD and genetic polymorphisms and found that MTHFR C677T and A1298C were independent risk factors for PPD [23], which may affect the incidence of PPD by changing the serum folic acid levels in women [24]. Some other genetic polymorphisms, such as 5-hydroxytryptamine transporter (5-HTT) and estrogen receptor (ESR), may also be related to increased susceptibility to PE and PPD [25-28]. Therefore, the abnormal expression of common genes such as MTHFR C677T, 5-HTT, and ESR may increase susceptibility to PPD in patients with $\mathrm{PE}$.

We must admit that there are some limitations in our study. As a retrospective study, it suffered from bias. First, our study only included the target population in our hospital, lacking data from other regions. Patients with severe PE might be more likely to follow up in postpartum clinic, and this may result in selection bias. Second, in our study, the subjects included pregnant women with PE only. Those with other obstetric complications such as gestational diabetes mellitus (GDM) and intrahepatic cholestasis of pregnancy (ICP) were excluded. But as we all know, preeclampsia is closely related to some other complications such as GDM, as they share common high-risk factors such as obesity and advanced maternal age [29]. Equally, GDM is also a risk factor for PPD [30]: for pregnant women with PE and other complications associated with $\mathrm{PE}$, if there was any change in the incidence of PPD; on the other hand, 93.3\% (84/90) of patients with PE in our hospital choose to deliver via cesarean section to prevent blood pressure elevation as well as to prevent cardiovascular and cerebrovascular accidents secondary to the pain of childbirth; however women who deliver via cesarean section show an increased risk of PPD [4]; in other areas where cesarean section rates are not so high, if the prevalence of PPD will decrease; these are all causes of system error and require multicenter research. Third our diagnosis of PPD depends on EPDS scores, which has been widely used as a screening tool throughout the world rather 
than as a tool for clinical diagnosis by psychiatrists. And we performed questionnaire survey to collect information on the last 7 days; this could also lead to information bias. Fourth, we did not investigate annual household income, maternal education, family support, and other factors of women involved in the experiment, since they are all risk factors for PPD. Fifth, since our study is the first to discuss the relationship between $\mathrm{PE}$ and $\mathrm{PPD}$, no other articles support our view. The several mechanisms we proposed are based on separate studies of PE and PPD. There were no targeted experiments to investigate whether there were differences in inflammatory factors and genetic changes in patients with PE and PPD. More research is required to explore the relationship between PE and PPD and its pathogenesis.

Despite some systematic errors, in our study, the prevalence of PPD in patients with PE was significantly higher than among normal women and, similarly, there was also an obvious difference in average score of EPDS between the two groups. So we identified PE as a risk factor for PPD. Youn, $\mathrm{H}$. et al. performed a study on the relationship between obstetric risk factors and PPD and similarly found that PE increases the incidence of PPD [12]. But more researches are needed to support our view

\section{Conclusion}

In our study, the prevalence of PPD was $26.67 \%$ (24/90) in patients with $\mathrm{PE}$, which was twice the prevalence of PPD among normal women (12.22\%). Patients who had PE had nearly 3-fold increased odds for PPD compared to normal women. Patients with severe PE have a more than 4-fold higher risk of screening positive for PPD. Several mechanisms, such as clinical symptoms, inflammation, and genetic changes, have been used to explain the relationship between $\mathrm{PE}$ and PPD, but experiments are needed to prove these relationships. Since PE is an independent risk factor for PPD, additional prevention efforts and support methods should be provided for women with PE to reduce the incidence of PPD.

\section{Data Availability}

The data used to support the findings of this study are included within the article.

\section{Conflicts of Interest}

The authors have no conflicts of interest to declare.

\section{Authors' Contributions}

Li Chen and Xiaodan Wang contributed equally to this article.

\section{Acknowledgments}

The authors would like to acknowledge support from the "111 program" of the Ministry of Education PRC and the State Administration of Foreign Experts Affairs PRC. This work was financially supported by the National Key Research and Development Program of Reproductive Health \& Major Birth Defects Control and Prevention (No. 2016YFC1000407), the
National Natural Science Foundation of China for Youth (81601304), the Key Program of International Cooperation of NSFC (81520108013), the Ph.D. Programs Foundation of the Ministry of Education of China (2013550311003), and the General Program of the National Natural Science Foundation of China (81471472).

\section{References}

[1] R. C. Kessler, P. Berglund, O. Demler et al., “The epidemiology of major depressive disorder: results from the National Comorbidity Survey Replication (NCS-R)," Journal of the American Medical Association, vol. 289, no. 23, pp. 3095-3105, 2003.

[2] B. N. Gaynes, N. Gavin, S. Meltzer-Brody et al., "Perinatal depression: prevalence, screening accuracy, and screening outcomes," Evidence Report/Technology Assessment (Summary), vol. 119, pp. 1-8, 2005.

[3] A. Gaillard, Y. Le Strat, L. Mandelbrot, H. Keita, and C. Dubertret, "Predictors of postpartum depression: Prospective study of 264 women followed during pregnancy and postpartum," Psychiatry Research, vol. 215, pp. 341-346, 2014.

[4] R.-H. Xie, J. Lei, S. Wang, H. Xie, M. Walker, and S. W. Wen, "Cesarean section and postpartum depression in a cohort of chinese women with a high cesarean delivery rate," Journal of Women's Health, vol. 20, no. 12, pp. 1881-1886, 2011.

[5] M. E. Silverman, L. Smith, P. Lichtenstein, A. Reichenberg, and S. Sandin, "The association between body mass index and postpartum depression: A population-based study," Journal of Affective Disorders, vol. 240, pp. 193-198, 2018.

[6] C. W. Swenson, J. A. DePorre, J. K. Haefner, M. B. Berger, and D. E. Fenner, "Postpartum depression screening and pelvic floor symptoms among women referred to a specialty postpartum perineal clinic," American Journal of Obstetrics and Gynecology, vol. 218, pp. 335.e331-335.e336, 2018.

[7] J. A. Hutcheon, S. Lisonkova, and K. S. Joseph, "Epidemiology of pre-eclampsia and the other hypertensive disorders of pregnancy," Best Practice \& Research. Clinical Obstetrics \& Gynaecology, vol. 25, pp. 391-403, 2011.

[8] American College of Obstetricians and Gynecologists and Task Force on Hypertension in Pregnancy, "Hypertension in pregnancy. Report of the american college of obstetricians and gynecologists' task force on hypertension in pregnancy," Obstetrics and Gynecology, vol. 122, no. 5, pp. 1122-1131, 2013.

[9] G. Lambert, J. F. Brichant, G. Hartstein, V. Bonhomme, and P. Y. Dewandre, "Preeclampsia: an update," Acta Anaesthesiologica Belgica, vol. 65, no. 4, pp. 137-149, 2014.

[10] A. Pálinkás, J. Sándor, M. Papp et al., "Associations between untreated depression and secondary health care utilization in patients with hypertension and/or diabetes," Social Psychiatry and Psychiatric Epidemiology, 2018.

[11] Z. Li, Y. Li, L. Chen, P. Chen, and Y. Hu, "Prevalence of depression in patients with hypertension: A systematic review and meta-analysis," Medicine, vol. 94, p. e1317, 2015.

[12] H. Youn, S. Lee, S. W. Han et al., "Obstetric risk factors for depression during the postpartum period in South Korea: a nationwide study," Journal of Psychosomatic Research, vol. 102, pp. 15-20, 2017.

[13] J. L. Cox, J. M. Holden, and R. Sagovsky, “Detection of postnatal depression. Development of the 10-item Edinburgh Postnatal Depression Scale," The British Journal of Psychiatry: The Journal of Mental Science, vol. 150, pp. 782-786, 1987. 
[14] Y. W. Xiujing Guo, Study on The Efficacy of The Edinburgh Postnatal Depression Scale in Puerperas in Chengdu, Chengdu, Sichuan University, 2007.

[15] M.-M. Li, Q.-F. Tang, and G.-Q. Zhang, "Application of assessment scale for postnatal depression in Chinese papers," Chinese Mental Health Journal, vol. 30, pp. 418-423, 2016.

[16] Y. Jonsson, M. Rubèr, L. Matthiesen et al., "Cytokine mapping of sera from women with preeclampsia and normal pregnancies," Journal of Reproductive Immunology, vol. 70, no. 1-2, pp. 83-91, 2006.

[17] A. Sharma, A. Satyam, and J. B. Sharma, "IL-10 and inflammatory markers (TNF-alpha, IL-6 and IL-8) in pre-eclamptic, normotensive pregnant and healthy non-pregnant women," American Journal of Reproductive Immunology (New York, N.Y. : 1989), vol. 58, pp. 21-30, 2007.

[18] C. Catarino, A. Santos-Silva, L. Belo et al., "Inflammatory disturbances in preeclampsia: relationship between maternal and umbilical cord blood," Journal of Pregnancy, vol. 2012, Article ID 684384, 10 pages, 2012.

[19] E. E. Accortt, C. D. Schetter, R. M. Peters, and A. E. CassidyBushrow, "Lower prenatal vitamin D status and postpartum depressive symptomatology in African American women: Preliminary evidence for moderation by inflammatory cytokines," Archives of Women's Mental Health, vol. 19, no. 2, pp. 373-383, 2016.

[20] H. Liu, Y. Zhang, Y. Gao, and Z. Zhang, "Elevated levels of Hs-CRP and IL-6 after delivery are associated with depression during the 6 months post partum," Psychiatry Research, vol. 243, pp. 43-48, 2016.

[21] G. M. Khandaker, R. M. Pearson, S. Zammit, G. Lewis, and P. B. Jones, "Association of serum interleukin 6 and C-reactive protein in childhood with depression and psychosis in young adult life: a population-based longitudinal study," JAMA Psychiatry, vol. 71, no. 10, pp. 1121-1128, 2014.

[22] X. Wu, K. Yang, X. Tang et al., "Folate metabolism gene polymorphisms MTHFR C677T and A1298C and risk for preeclampsia: a meta-analysis," Journal of Assisted Reproduction and Genetics, vol. 32, no. 5, pp. 797-805, 2015.

[23] Y.-d. Li, H.-y. Yue, and H. Li, "Effect of folic acid intake during pregnancy and MTHFR polymorphism on postpartum depression," Chinese Journal of Woman and Child Health Research, vol. 28, pp. 632-635, 2017.

[24] J. Yan, Y. Liu, L. Cao, Y. Zheng, W. Li, and G. Huang, "Association between duration of folic acid supplementation during pregnancy and risk of postpartum depression," Nutrients, vol. 9, no. 11, p. 1206, 2017.

[25] A. C. Bolte, H. P. Van Geijn, and G. A. Dekker, "Pathophysiology of preeclampsia and the role of serotonin," European Journal of Obstetrics \& Gynecology and Reproductive Biology, vol. 95, no. 1, pp. 12-21, 2001.

[26] Y. Yang, M. Fang, X. Du, and Z. Hu, "Lucky gene 5-HTTLPR and postpartum depression: A systematic review," Neuroendocrinology Letters, vol. 38, pp. 316-320, 2017.

[27] G. Yin, X. Zhu, C. Guo et al., "Differential expression of estradiol and estrogen receptor alpha in severe preeclamptic pregnancies compared with normal pregnancies," Molecular Medicine Reports, vol. 7, no. 3, pp. 981-985, 2013.

[28] J. Ryan, J. Scali, I. Carrière et al., "Estrogen receptor alpha gene variants and major depressive episodes," Journal of Affective Disorders, vol. 136, no. 3, pp. 1222-1226, 2012.

[29] Y. Yogev, O. Langer, L. Brustman, and B. Rosenn, "Preeclampsia and gestational diabetes mellitus: does a correlation exist early in pregnancy?" The Journal of Maternal-Fetal \& Neonatal Medicine, vol. 15, no. 1, pp. 39-43, 2004.

[30] A. Ruohomäki, E. Toffol, S. Upadhyaya et al., "The association between gestational diabetes mellitus and postpartum depressive symptomatology: A prospective cohort study," Journal of Affective Disorders, vol. 241, pp. 263-268, 2018. 


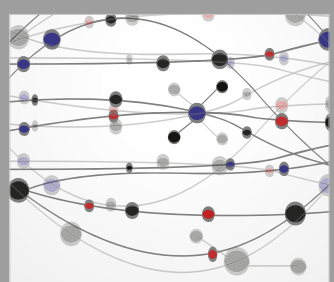

The Scientific World Journal
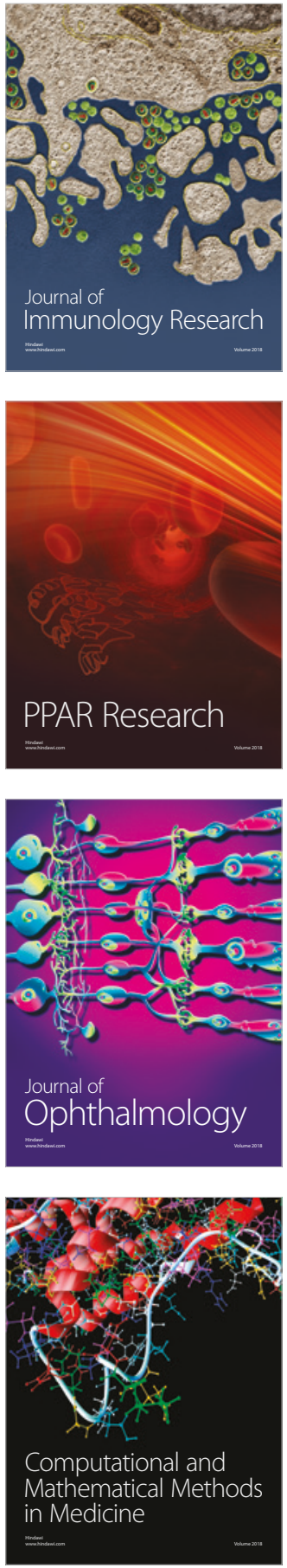

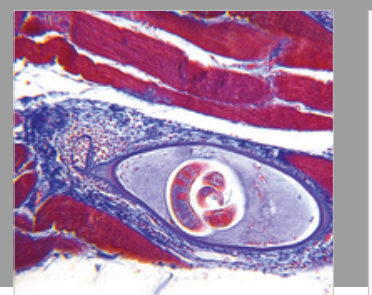

Gastroenterology Research and Practice

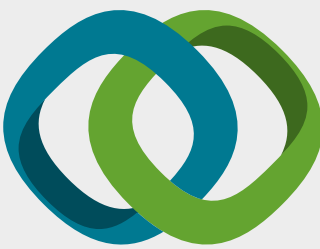

\section{Hindawi}

Submit your manuscripts at

www.hindawi.com
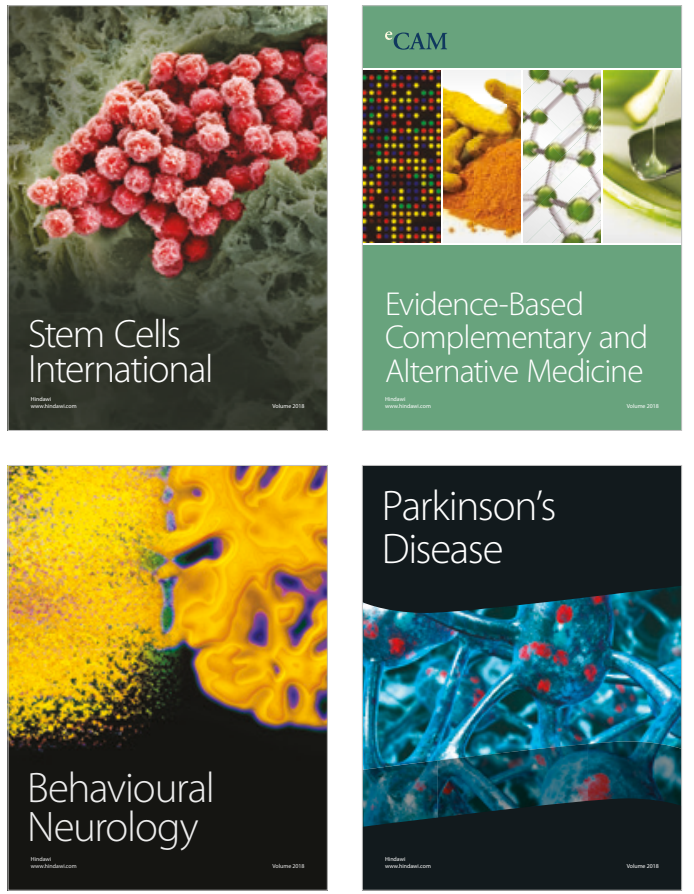

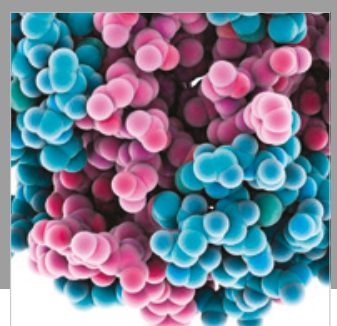

ournal of

Diabetes Research

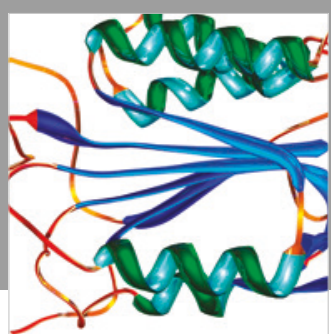

Disease Markers
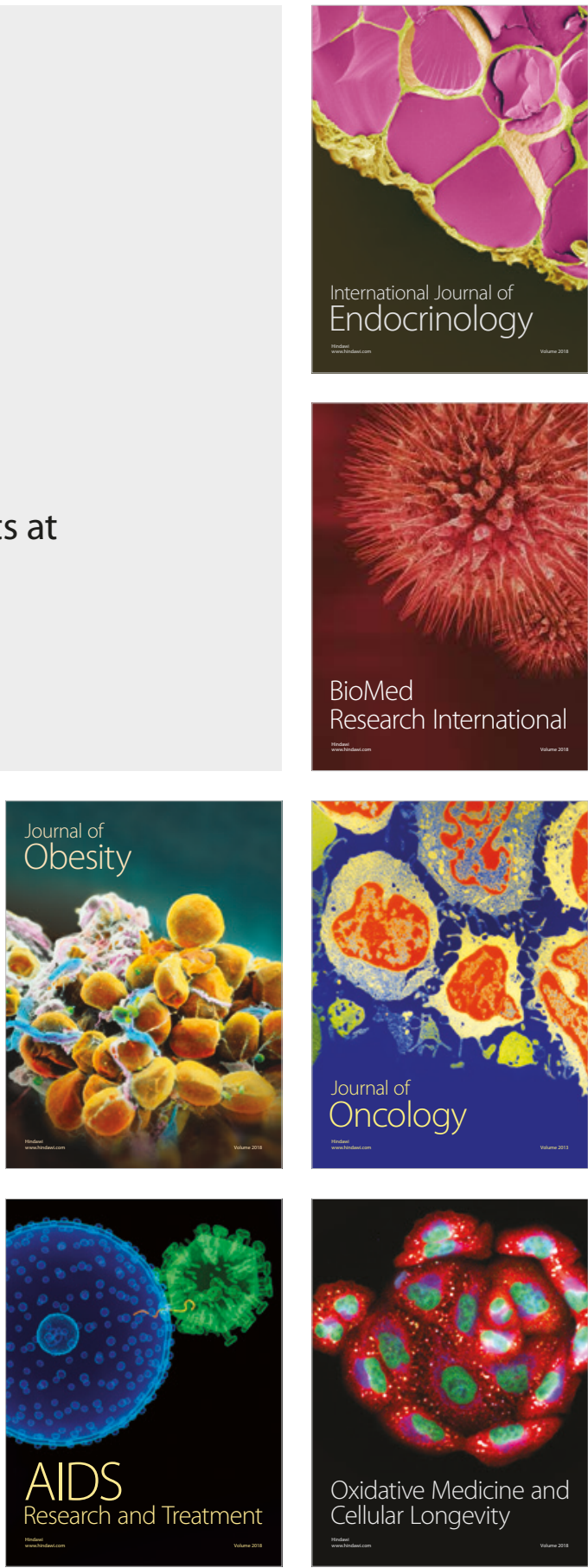